\title{
Silencing of the human TERT gene by RNAi inhibits A549 lung adenocarcinoma cell growth in vitro and in vivo
}

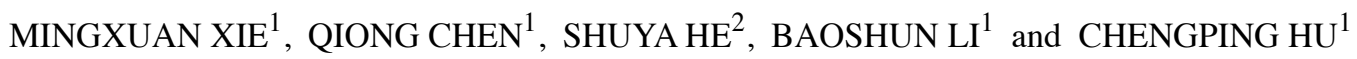 \\ ${ }^{1}$ Department of Respiratory Medicine, Department of Gerontology, Xiangya Hospital, \\ Central South University, Changsha 410008; ${ }^{2}$ Department of Biochemistry and \\ Molecular Biology, University of South China, Hengyang 421001, P.R. China
}

Received May 10, 2011; Accepted June 14, 2011

DOI: $10.3892 /$ or.2011.1383

\begin{abstract}
Human telomerase reverse transcriptase (hTERT) is the catalytic subunit and the activity determinant factor of the telomerase enzyme which maintains the length of human chromosomes. In recent years it has become an attractive molecular target for cancer gene therapy. In the present study, we show that hTERT siRNA effectively suppressed the expression of hTERT mRNA and hTERT protein levels, reduced telomerase activity, and induced apoptosis of A549 lung adenocarcinoma cells $(\mathrm{P}<0.05)$. In vivo, tumors treated with the hTERT siRNA were of reduced sizes, indicating that the hTERT siRNA also reduced the tumorigenic potential of lung adenocarcinoma cells $(\mathrm{P}<0.05)$. These results demonstrate that hTERT siRNA can cause effective suppression of telomerase and lead to apoptosis in A549 lung adenocarcinoma cells. hTERT siRNA may, therefore, be a strong candidate for highly selective therapy for chemoprevention and treatment of lung adenocarcinoma.
\end{abstract}

\section{Introduction}

Telomerase, a ribonucleoprotein holoenzyme, maintains the length of telomeres which protect the chromosomes from DNA degradation, end-to-end fusions, rearrangements and chromosome loss at the ends of eukaryotic linear chromosomes. hTERT, the catalytic subunit of the telomerase complex, maintains the length of telomeres by reverse transcription and addition of TTAGGG repeats onto the telomeric ends of the chromosomes (1). The cellular activity of telomerase is determined by the presence or absence of hTERT and hTERT is the major determinant of telomerase activity (2). Human normal somatic cells have no telomerase activity. During each cell division, a portion

Correspondence to: Dr Qiong Chen, Department of Respiratory Medicine, Department of Gerontology, Xiangya Hospital, Central South University, 87 Xiangya Road, Changsha, Hunan 410008, P.R. China

E-mail: qiongch@yahoo.com.cn

Key words: human telomerase reverse transcriptase, lung adenocarcinoma, RNAi, siRNA, telomerase of terminal DNA repeats may be lost with each cycle of DNA replication. When telomeres reach a critical length, the cells send out a signal to stop chromosome duplication and cellular division, and cellular senescence and apoptosis occur (3). But the majority of cancer cell lines and tumors have high telomerase activities, mainly by enhancing the expression of hTERT (4). Research shows a high expression of the hTERT gene in $90 \%$ malignant cancers, while low or undetectable levels in most normal tissue cells. Numerous studies have demonstrated that telomerase activity is present in almost all tumor samples (5), and our previous study has also shown that telomerase activity could be detected in $75 \%$ non-small cell lung cancer (NSCLC) specimens (6). Moreover, Tomlinson et al (7) found that the trafficking of hTR to both telomeres and Cajal bodies, where telomerase is active and is not observed in primary cells, depends on hTERT. Furthermore, telomerase seems to exert its anti-apoptotic effects via extratelomeric mitochondria and the caspase pathway $(8,9)$. Therefore, inhibition of hTERT results in an inability of tumor cells to maintain telomeres and in loss of extratelomeric anti-apoptotic roles thus leading to tumor cell apoptosis. In recent years, hTERT has become an attractive molecular target for cancer therapy.

Reverse transcriptase inhibitors, antisense oligonucleotides and RNA interference (RNAi) all induce telomeric instability and apoptosis of tumor cells. A reverse transcriptase inhibitor, because of its characteristics of a nucleoside analogue, is not an ideal antitumor drug. Its inhibitory activity on telomerase is weak; moreover, it has inhibitory effects on other DNA and RNA polymerases, which give rise to a multitude of side effects (10). Antisense oligonucleotides are not stable in vivo (11). Though chemical modification can improve its stability, it weakens its affinity to complementary strand and facilitates its binding to some other proteins, therefore causing side effects which limit its clinical application (12).

RNAi is the process by which double-stranded RNA (dsRNA) targets mRNA for destruction in a sequence-dependent manner. RNAi is in fact a natural process, and this is best exemplified by the discovery of naturally encoded structural hairpin RNA molecules that are called microRNAs (miRNAs), which are now known to play extremely important roles in regulating gene expression at the post-transcriptional level $(13,14)$. The mechanism of RNAi is initiated with processing of dsRNA into approximately $21-23$ bp fragments that hybridize with the 
target mRNA. An enzyme called Dicer processes dsRNA into the short fragments (15). These small cleavage products are then incorporated into a larger, multi-protein RNA-induced silencing complex (RISC), which simultaneously scans the complementary mRNA sequence for homology to the small, now unwound, RNA fragment and then promotes the destruction of the mRNA through an enzymatic activity integral to the complex (16). Massive research and wide applications of this technique are motivated by the hypothesis that effectors of RNAi can be developed into effective drugs for treating malignancies as well as a tool for detecting mutant genes. Up to date there are only a few in vitro or in vivo studies on the inhibitory effect of hTERT siRNA on lung adenocarcinoma. We systematically studied the impact of the 21-bp hTERT siRNA on the expression of hTERT mRNA, hTERT protein, telomerase activity and the apoptosis of the lung adenocarcinoma A549 cells, and further explored the inhibitory effect of hTERT siRNA on the growth of athymic mouse xenografts of lung adenocarcinoma in vivo.

\section{Materials and methods}

Cell culture. Human lung carcinoma A549 cells (Cell Culture Center of Central South University) were grown in Dulbecco's modified Eagle's medium (DMEM), supplemented with $10 \%$ fetal bovine serum, $100 \mathrm{U} / \mathrm{ml}$ penicillin and $100 \mu \mathrm{g} / \mathrm{ml}$ streptomycin at $37^{\circ} \mathrm{C}$ and $5 \% \mathrm{CO}_{2}$. Cells were digested and passaged by $0.25 \%$ trypsin every two or three days to maintain exponential growth. All cell culture materials were purchased from Invitrogen, USA.

Transfection. A549 cells were divided into the hTERT siRNA group, control siRNA group and liposome group according to different treatments. The hTERT siRNA group was transfected with hTERT siRNA (100 nmol/l) and Lipofectamine ${ }^{\mathrm{TM}} 2000$ (Invitrogen) $(2 \mu \mathrm{g} / \mathrm{ml})$; the control siRNA group with control siRNA (100 nmol/l) and Lipofectamine $2000(2 \mu \mathrm{g} / \mathrm{ml})$; and the liposome group with Lipofectamine $2000(2 \mu \mathrm{g} / \mathrm{ml})$. Transfection of siRNA was carried out according to the Lipofectamine 2000 manufacturer's instructions. Cells $\left(1.5 \times 10^{5} /\right.$ well $)$ were seeded in a 6-well plate and 5 wells were plated for each group. The cells were allowed to grow in antibiotic-free complete growth media for $24 \mathrm{~h}$ before transfection. The cells were about $40-50 \%$ confluent at the time of transfection.

Selection and synthesis of siRNA sequences targeting hTERT $m R N A$. Three 21-mer siRNA sequences directed against the hTERT mRNA (GenBank AB085628) were designed by an online commercial software (Applied Biosystems; Austin, TX, USA) and by referring to published reports (17-19), and were then submitted to BLAST to avoid an off-target effect. siRNA sequences used in the study and their target siRNA sites on the hTERT mRNA were as follows: siRNA-1 (1755-1773), 5'-GC AUUGGAAUCAGACAGCAUU-3'; siRNA-2 (1876-1894), 5'-UGAUUUCUUGUUGGUGACAdTdT-3'; siRNA-3 (19832001), 5'-GAACGUUCCGCAGAGAAAATT-3'. One control siRNA was used, 5'-GAACGCGUUCAGUAAAACGUU-3'. In vitro transcription of siRNA was carried out using the In vitro Transcription T7 kit (for siRNA Synthesis) (Takara Bio Inc., Japan) according to the manufacturer's protocol.
After $24 \mathrm{~h}$ of transfection, expressions of hTERT mRNA and protein were measured. Eventually we chose the siRNA-1, which had the most obvious down-regulating effect among the three siRNAs, as the experimental siRNA to conduct the subsequent interfering experiments.

Analysis of hTERT mRNA by RT-PCR. Total hTERT mRNA was isolated by TRIzol (Molecular Research Center, OH, USA) every $24 \mathrm{~h}$ for three days. The cDNA strand was synthesized using a reverse transcription kit (Fermentas, MBI, USA) according to the manufacturer's instructions. PCR primers were as follows: hTERT (20), 5'-TCTACCGGAAGAGTGTCTG GAGCAA-3' (forward) and 5'-GCTCCCACGACGTAGTCC ATGTTCA-3' (reverse), amplicon, 202 bp; $\beta$-actin (21), 5'-CCA AGGCCAACCGCGAGAAGATGAC-3' (forward) and 5'-AGG GTACATGGTGGTGCCGCCAGAC-3' (reverse); amplicon, $540 \mathrm{bp}$. Reaction parameters were $94^{\circ} \mathrm{C}$ for $5 \mathrm{~min} ; 94^{\circ} \mathrm{C}$ for $40 \mathrm{sec}, 60^{\circ} \mathrm{C}$ for $30 \mathrm{sec}$, and $72^{\circ} \mathrm{C}$ for $45 \mathrm{sec}$, for 30 cycles; $72^{\circ} \mathrm{C}, 10 \mathrm{~min}$. The inhibitory rate $(\%)$ of hTERT mRNA was calculated by the relative intensity ratio hTERT/ $\beta$-actin according to the following formula (20):

$1-\frac{\text { relative intensity ratio of hTERT siRNA group hTERT mRNA }}{\text { relative intensity ratio of liposome group hTERT mRNA }} \times 100$

Analysis of hTERT protein by Western blot analysis. Cell extract lysates $(100 \mu \mathrm{g})$ were loaded on polyacrylamide gels and subjected to SDS gel electrophoresis, then transferred to a nitrocellulose membrane. The membrane was treated with blocking solution ( $5 \%$ non-fat milk, and $0.02 \%$ sodium azide in phosphate-buffered saline) for $1 \mathrm{~h}$ at room temperature. The membrane was incubated overnight at $4^{\circ} \mathrm{C}$ with the primary antibody, mouse anti-human hTERT antibody (1:1000 dilution, Abcam). The membrane was then incubated for $1 \mathrm{~h}$ at room temperature with rabbit anti-mouse secondary antibody, followed by detection with chemiluminescent reagents (ECL kit, Amersham, USA). The inhibitory rate (\%) of the hTERT protein was calculated according to the following formula:

$$
1-\frac{\text { hTERT protein positive rate of the hTERT siRNA group }}{\text { hTERT protein positive rate of the liposome group }} \times 100
$$

Analysis of telomerase activity by TRAP-ELISA. Telomerase activity was determined every $24 \mathrm{~h}$ for three days after transfection by TeloTAGGG Telomerase PCR ELISA ${ }^{\text {PLUS }}$ (Roche, USA). Briefly, $1.5 \times 10^{5}$ cells were centrifuged for $10 \mathrm{~min}$ at $4000 \mathrm{rpm}$ and the sediments were suspended with lysis buffer (10 mM EGTA, 10 mM EDTA, 62.5 mM Tris-HCl, pH 6.8, $2 \%$ sodium dodecyl sulfate) on ice for $30 \mathrm{~min}$. The supernatant was used as a TRAP template after centrifuging at $14,000 \mathrm{rpm}$ for $20 \mathrm{~min}$. The reaction mixture was incubated at $25^{\circ} \mathrm{C}$ for $30 \mathrm{~min}$, and then PCR amplification was performed for 35 cycles of $94^{\circ} \mathrm{C}$ for $30 \mathrm{sec}$ and $59^{\circ} \mathrm{C}$ for $60 \mathrm{sec}$ in each. The PCR product was initially mixed with hybridization solution, incubated at $37^{\circ} \mathrm{C}$ for $1 \mathrm{~h}$, followed by washing, incubation for another $30 \mathrm{~min}$, chromogenized and the absorbance was 

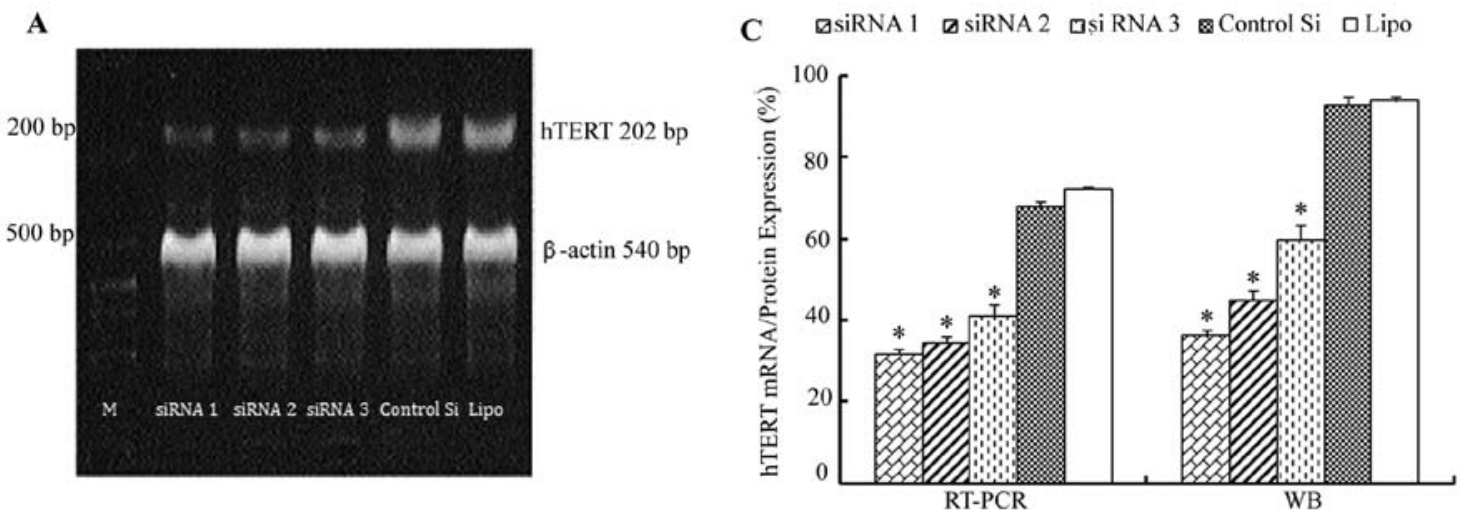

B

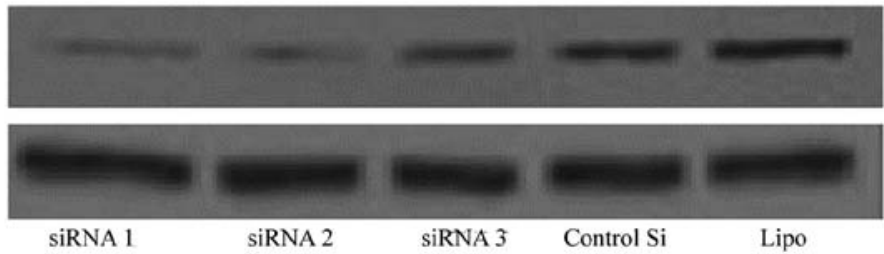

Figure 1. Selection of siRNAs. The three hTERT siRNAs, one control siRNA and a liposome vector were transiently transfected into the lung adenocarcinoma cell lines A549 respectively and incubated for $24 \mathrm{~h}$. (A) hTERT mRNA expression. (B) hTERT protein expression. (C) Statistical analysis. ("P $<0.05$ compared with the liposome group).

detected according to the manufacturer's instruction. Relative telomerase activities (RTA) within different samples in an experiment were obtained using the following formula:

$$
\mathrm{RTA}=100 \times\left(\mathrm{A}_{\mathrm{S}}-\mathrm{A}_{\mathrm{S}, \mathrm{O}}\right) \times \mathrm{A}_{\mathrm{TS} 8, \mathrm{IS}} / \mathrm{A}_{\mathrm{S}, \mathrm{IS}} \mathrm{x}\left(\mathrm{A}_{\mathrm{TS} 8}-\mathrm{A}_{\mathrm{TS} 8,0}\right)
$$

where $\mathrm{A}_{\mathrm{S}}$, absorbance of sample; $\mathrm{A}_{\mathrm{S}, \mathrm{O}}$, absorbance of negative control; $\mathrm{A}_{\mathrm{S}, \mathrm{IS}}$, absorbance of internal standard of the sample; $\mathrm{A}_{\mathrm{TS} 8}$, absorbance of control template; $\mathrm{A}_{\mathrm{TS} 8,0}$, absorbance of lysis buffer; $A_{\mathrm{TS} 8, \mathrm{IS}}$, absorbance of internal standard of the control template. Inhibition of telomerase activity was calculated according to previous methods.

Analysis of cell apoptosis by flow cytometry. Apoptosis was analyzed with Annexin V and PI double staining by a flow cytometer every 3 days for 15 days after transfection. To double-label the cells, $10^{5}$ trypsinized cells were washed twice with PBS and centrifuged at $2000 \mathrm{rpm}$ for $5 \mathrm{~min}$. Resuspended cells in $500 \mu \mathrm{l}$ of binding buffer were stained by adding $5 \mu \mathrm{l}$ of Annexin V-FITC and $5 \mu \mathrm{l}$ PI. After a 15-min incubation at room temperature, fluorescence was measured by a flow cytometry using a FACS Calibur with a $488 \mathrm{~nm}$ laser line and the CellQuest software (Becton-Dickinson). A total of $10^{4}$ cells were analyzed in each assay to create a cytogram.

Mouse xenograft model of lung adenocarcinoma. The study was approved by the Animal Research Committee of the Central South University and carried out in accordance with the National Institutes of Health Guide for the Care and Use

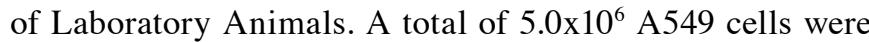
inoculated in $0.1 \mathrm{ml}$ Opti-MEM I reduced serum medium through a 24-gauge needle injected into the lower flank of Balb/c-nu mice, a kind of athymic nude mice (age, 4-6 weeks; body weight, 18-22 g) (Experimental Animal Department of
Central South University). After 3 weeks, when the tumors had reached an average volume of approximately $50-60 \mathrm{~mm}^{3}$, the tumor-bearing nude mice were divided into two groups, 5 mice each. The hTERT siRNA group was treated with hTERT siRNA plus Lipofectamine 2000 and the control group with Lipofectamine 2000 alone. The final concentration of Lipofectamine 2000 was $2 \mu \mathrm{g} /$ tumor and that of hTERT siRNA was $5.0 \mu \mathrm{g} /$ tumor. hTERT siRNAs were used after dilution with Opti-MEMI Reduced Serum Medium. These reagents were injected into the tumors 2 times a week for 4 weeks.

Analysis of the tumor. The length (L) and width (W) of the tumors were measured at intervals of 7 days with digital calipers during treatment. On the 28th day, tumor diameters and weights were determined after nude mice were sacrificed and the normal tissue on the tumor was cleared. The tumor volume in $\mathrm{mm}^{3}$ was calculated by the formula (22):

$$
\text { Volume }=\mathrm{L} \mathrm{x} \mathrm{W}^{2} / 2
$$

and the inhibitory rate was calculated by the formula:

$$
\text { Inhibitory rate }(\%)=(1-\mathrm{V} / \mathrm{v}) \times 100 \%
$$

where V, volume change in the hTERT siRNA group; v, volume change in the control group. Twenty-eight days after treatment, tumor tissue was fixed with phosphate buffered $10 \%$ formalin and embeded in paraffin. Routine histology (hematoxylin and eosin staining) was performed to compare the difference of basic histomorphological features between the two groups.

Analysis of hTERT $m R N A$, hTERT protein and telomerase activity of the tumor. The expression of hTERT mRNA and protein and telomerase activity of the tumor were assayed by 
A

$24 \mathrm{~h}$

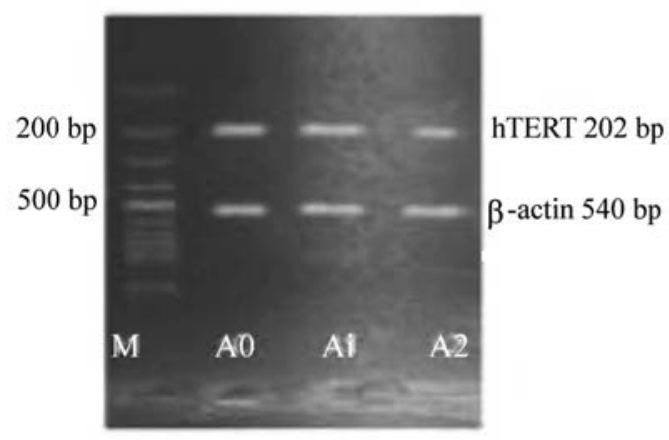

C

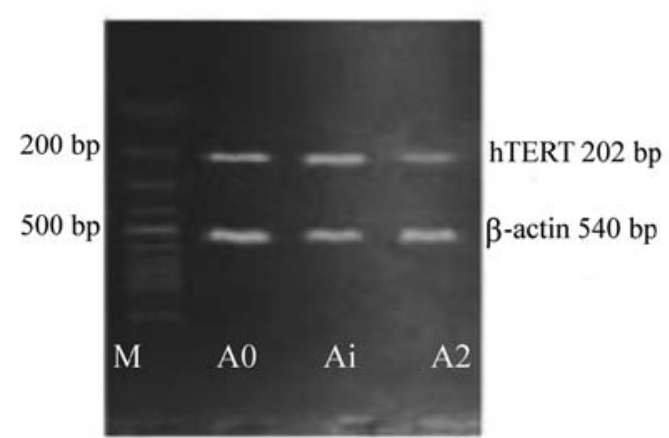

B

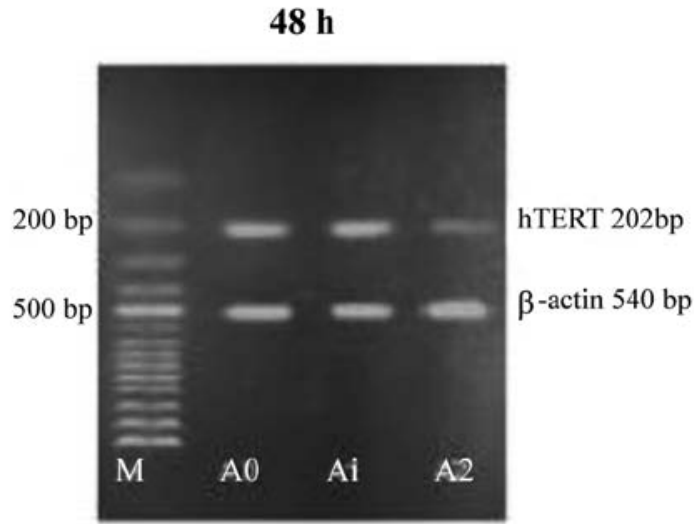

D

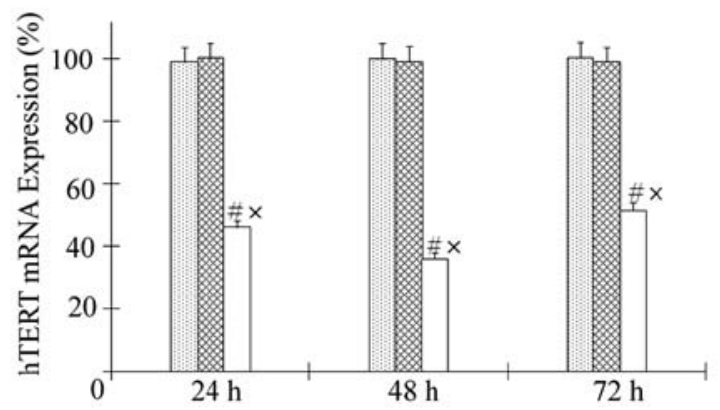

Figure 2. Reduced expression of hTERT mRNA by hTERT. The concentration and purity of mRNA were assayed by an ultraviolet spectrophotometer and the inhibitory rates were calculated every $24 \mathrm{~h}$ for three days after transfection. M, Marker; A0, liposome group; A1, control siRNA group; A2, hTERT siRNA group. ${ }^{\text {}} \mathrm{P}<0.05$ compared to the liposome group; ${ }^{\times} \mathrm{P}<0.05$ compared to the hTERT siRNA group.

RT-PCR, Western blot analysis and TRAP-ELISA, respectively, which have been described previously.

Statistical analysis. The measurement data were presented with mean \pm SD. Multiple comparisons were performed with the SNK-test and comparisons between two means were carried out with the t-test. Correlation analysis was also used to detect the relationship between tumor volume change and tumor telomerase activity. Significance is defined as $\mathrm{P}<0.05$.

\section{Results}

Selection of siRNAs to down-regulate hTERT. To measure the three siRNAs against the telomerase gene, we conducted RT-PCR and Western blot analyses after $24 \mathrm{~h}$ to identify the best siRNA for our subsequent experiments. As shown in Fig. 1A, the hTERT mRNA expressions were $31.83,34.43,41.24 \%$ in the three siRNA groups, $67.82 \%$ in the control siRNA group, and $72.08 \%$ in the liposome group, respectively compared to their $\beta$-actin mRNA expressions. As shown in Fig. 1B, the hTERT protein expressions were $36.27,44.98,59.70,92.83$ and $93.77 \%$ respectively, compared to their $\beta$-actin protein expressions. The three siRNA can effectively reduce the expressions of hTERT mRNA and protein so that they perfectly exclude the possibility of an off-target effect which the strategy of siRNA may sometimes have. Eventually, we chose siRNA-1 (later referred as hTERT siRNA) to conduct our subsequent experiments as it showed the most obvious knockdown effect.
hTERT $m R N A$ and protein expression. The hTERT siRNA was transiently transfected into the A549 lung adenocarcinoma cell line. The inhibitory rates were calculated every $24 \mathrm{~h}$ for three days after transfection. For RNA levels, as shown in Fig. 2, the inhibitory rates were 53.62, 64.03 and 49.69\%, respectively. For protein levels, as shown in Fig. 3, the inhibitory rates were $29.15,39.48$ and $17.85 \%$, respectively. The inhibition of hTERT mRNA and protein expression reached a maximum level at $48 \mathrm{~h}$ and continued up to $72 \mathrm{~h}$ after transfection. The control siRNA group and the liposome group showed no effects on the hTERT mRNA or protein levels. The results indicate that hTERT siRNA was also successful in knocking down the hTERT gene and the effect of the hTERT siRNA was specific and effective.

Telomerase activity. Telomerase activity as determined by TRAP-ELISA and the relative telomerase activities were assayed every $24 \mathrm{~h}$ for three days after transfection. As shown in Fig. 4A, the inhibitory rates were 54.47, 69.30 and 44.27\%, respectively. The inhibitory rate reached a maximum at $48 \mathrm{~h}$ and persisted $72 \mathrm{~h}$ after transfection. The control siRNA group and the liposome group showed no effects on telomerase activity. The difference was not significant. These results suggest that hTERT siRNA transfection inhibited the telomerase activity.

Induction of apoptosis following hTERT siRNA treatment. Apoptosis was analyzed by flow cytometry every 3 days for 15 
A

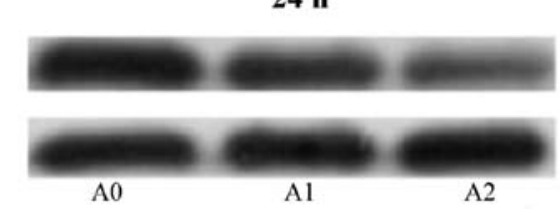

C

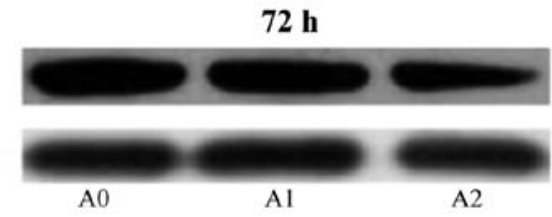

B

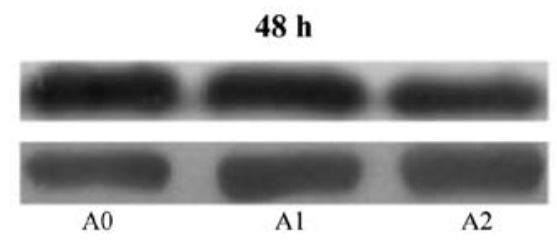

D

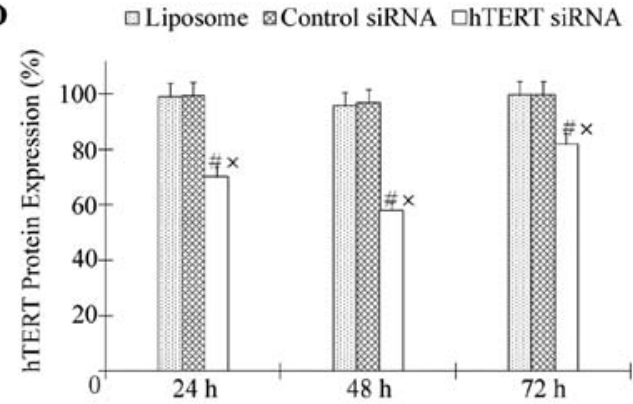

Figure 3. Reduced expression of hTERT protein by hTERT siRNA. The protein levels were assayed by Western blot analysis and the inhibitory rates were calculated every $24 \mathrm{~h}$ for 3 days after transfection. M, Marker; A0, liposome group; A1, control siRNA group; A2, hTERT siRNA group. "P<0.05 compared with the liposome group; ${ }^{\times} \mathrm{P}<0.05$ compared with the hTERT siRNA group.

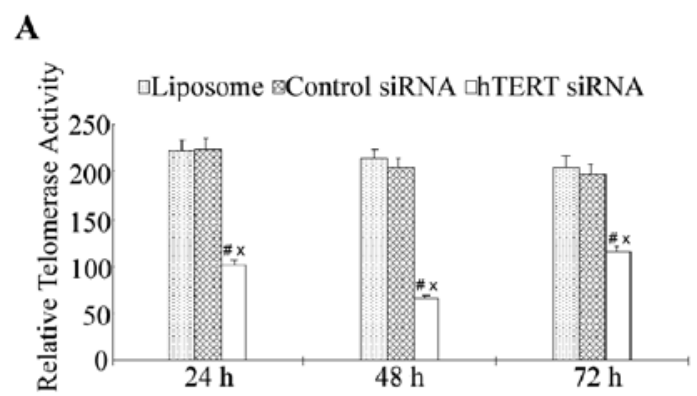

$\mathbf{B}$

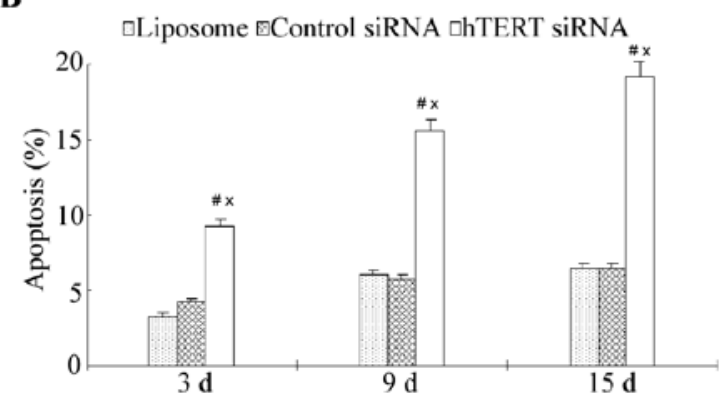

Figure 4. (A) hTERT siRNA inhibits the telomerase activity of A549 human lung adenocarcinoma cells. Telomerase activities of each group were determined by TRAP-ELISA every $24 \mathrm{~h}$ for three days after transfection and the relative telomerase activities were analyzed. (B) hTERT siRNA induced early apoptosis of A549 human lung adenocarcinoma cells. Apoptosis was analyzed by Annexin V-FITC/PI double-staining flow cytometry every 3 days for 15 days after transfection. $\left({ }^{\#} \mathrm{P}<0.05\right.$ compared with the liposome group; ${ }^{\mathrm{P}}<0.05$ comparison with hTERT siRNA group).

days after the first transfection. Transfection was performed twice a week to guarantee its silencing effect. Similarly to the results presented in Figs. 4B and 5, Annexin V-FITC/ PI double-staining showed that the early apoptotic rates for the hTERT siRNA transfected group were 17.63, 18.16 and $23.07 \%$ on days 3,9 and 15 , respectively, while those of the control group were $12.96,11.28$ and $9.29 \%$, respectively. The apoptotic rates gradually increased from the third day to the fifteenth day after transfection. The control siRNA group and the liposome group showed comparatively similar apoptosis rates of lung adenocarcinoma cells. The difference was not significant. These results suggest that hTERT siRNA can induce the apoptosis of A549 human lung adenocarcinoma cells.

Mouse xenograft model of lung adenocarcinoma. A549 cells $\left(5.0 \times 10^{6}\right)$ were injected into the flank of nude mice. After 3 weeks, visible tumors had developed at the injection sites. As shown in Fig. 6A, the tumor weight of control group was $344 \pm 143 \mathrm{mg}$ which was significantly bigger than hTERT siRNA group which was $158 \pm 97 \mathrm{mg}(\mathrm{t}=2.397, \mathrm{P}<0.05)$. As shown in Fig. 6B and D, the average tumor volume of control group was $766.7 \pm 339.2 \mathrm{~mm}^{3}$ which was significantly higher than hTERT siRNA group which was $330.0 \pm 97.1 \mathrm{~mm}^{3} 4$ weeks after transfection $(\mathrm{t}=2.364, \mathrm{P}<0.05)$. As shown in Fig. 6C, the average tumor volume change of control group was $712.1 \pm 311.0 \mathrm{~mm}^{3}$ which was significantly higher than hTERT siRNA group which was $254.4 \pm 231.9 \mathrm{~mm}^{3}$ after transfection $(\mathrm{t}=2.368, \mathrm{P}<0.05)$. The inhibition rate reached $64.28 \%$. The tumor volume curve is shown in Fig. 6E. It is self-evident that hTERT siRNA potently suppressed the growth of nude mice xenograft of lung adenocarcinoma. For histological differences, the hTERT siRNA group showed less pathological nuclear divisions and polykaryocytes, smallersized cells distributing into the form of nest and trab, some structures similar to gland alveolus in some regions and more interstitial fibrous tissues than the control group (Fig. 6F). It showed that hTERT siRNA alleviated the heteromorphism of xenograft tumor tissue.

hTERT mRNA and protein expression of lung adenocarcinoma xenografts. RT-PCR revealed that the relative amount of 

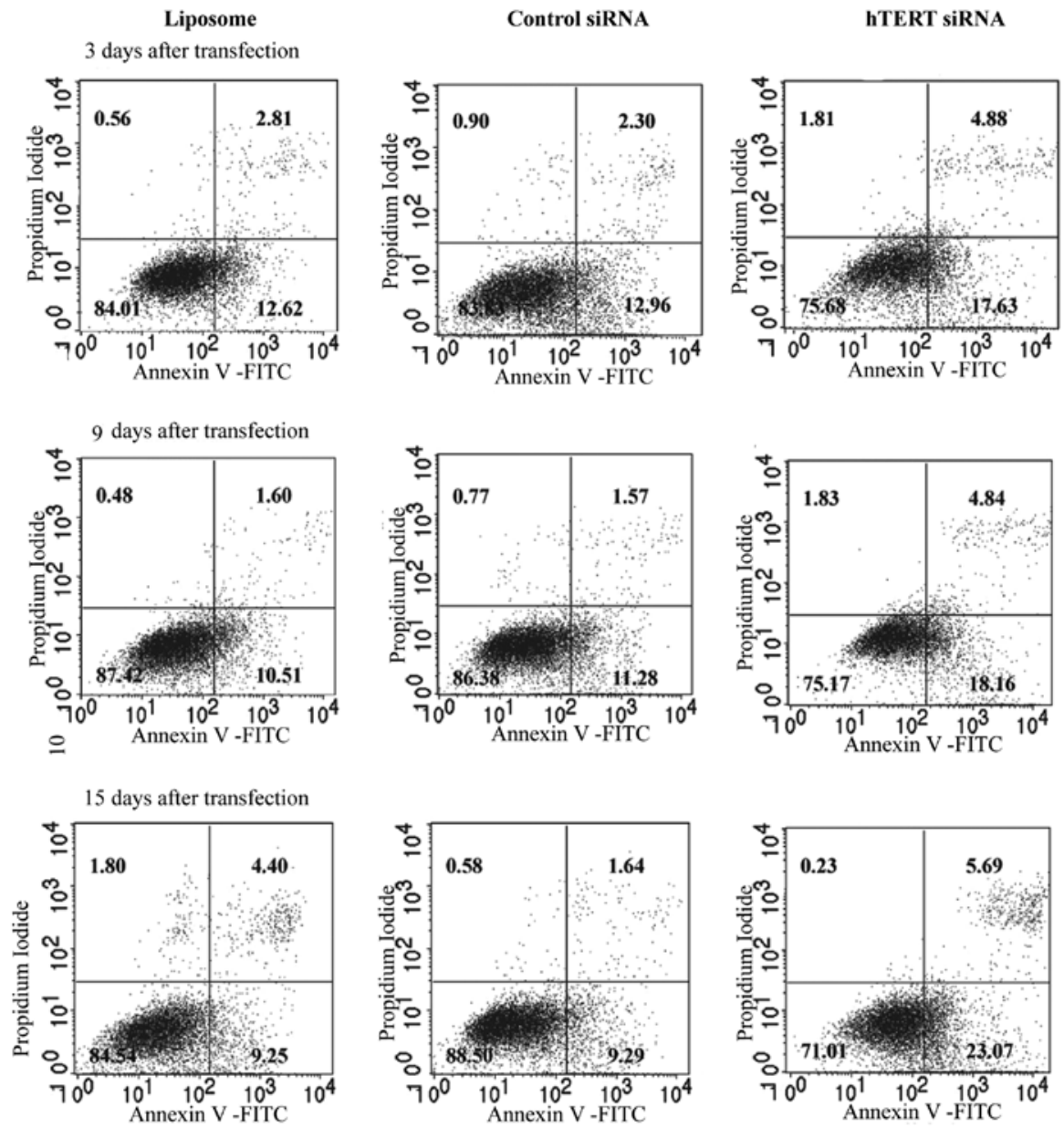

Figure 5. hTERT siRNA induces early apoptosis of human lung adenocarcinoma A549 cells. Detailed pictures and statistical data for Annexin V-FITC/PI double-staining flow cytometer results.

hTERT mRNA expression of the control group tumor was $99.06 \pm 1.84 \%$, which was significantly higher than that in the hTERT siRNA group which was $54.47 \pm 4.33 \% 4$ weeks after transfection $(\mathrm{t}=21.179, \mathrm{P}<0.05)$ (Fig. 7A and $\mathrm{B})$. The highest inhibitory rate reached $45.01 \%$. As shown in Fig. $7 \mathrm{C}$ and D, immunohistochemistry assay with CMIAS revealed that the hTERT protein expression in the control group tumor was $77.4 \pm 4.7 \%$ which was significantly higher than that of the hTERT siRNA group which was $38.0 \pm 4.9 \% 4$ weeks after transfection $(\mathrm{t}=12.901, \mathrm{P}<0.05)$. By using TeloTAGGG Telomerase PCR ELISA ${ }^{\text {PLUS }}$, the average telomerase activity of the control group tumor was $210.06 \pm 30.06$ which was significantly higher than that of the hTERT siRNA group which was $102.38 \pm 8.274$ weeks after transfection $(\mathrm{t}=7.727, \mathrm{P}<0.05)$. The relative telomerase activity was $51.26 \%$ (Fig. 7E). It is suggested that hTERT siRNA potently suppressed the expression of hTERT mRNA and protein, and telomerase activity in lung adenocarcinoma xenograft. As shown in Fig. 7F, the lower the telomerase activities in the xenografts, the slower its growth. The relative telomerase activity of the xenografts positively correlated to tumor volume change $(\mathrm{r}=0.932, \mathrm{P}<0.05)$.

\section{Discussion}

A rational design for selecting potent siRNAs can facilitate functional gene knockdown $(18,23)$. Our results showed that
hTERT siRNA could down-regulate the expression of hTERT mRNA and protein in A549 human lung adenocarcinoma. The inhibitory effect was most evident $48 \mathrm{~h}$ after transfection. In the study of Xia et al five double-stranded siRNAs targeting coding and non-coding regions of the hTERT gene were devised and transfected in the HepG2 human hepatocellular carcinoma cell line, and in accordance with our data the results showed that hTERT siRNA can inhibit the expression of hTERT mRNA and protein; the highest inhibitory rates at 24 and $48 \mathrm{~h}$ after transfection were 70.7 and $82.5 \%$ (20). We further observed the effect of hTERT siRNA on telomerase activity of A549 human lung adenocarcinoma cells. Our results showed that telomerase activity in human lung adenocarcinoma cells can be inhibited by hTERT siRNA. This effect was most evident $48 \mathrm{~h}$ after transfection, consistent with the down-regulation of hTERT mRNA and protein. Kosciolek et al (24) demonstrated that hTERT siRNA can suppress a variety of adenocarcinoma cells such as the HCT-15 colon carcinoma, HeLa cervical carcinoma, NCI H123 lung carcinoma and A431 epidermoid carcinoma, with a maximum inhibitory rate of $65 \%$. Shammas et al (25) designed siRNAs against two different regions of the telomerase gene in Barrett's adenocarcinoma SEG-1 cells. The results showed that hTERT protein level had decreased by $95 \%$ in the siRNA group than in the control group; telomerase activity had decreased by $77 \pm 3 \%$ at $24 \mathrm{~h}$ after transfection and had been completely inhibited at $72 \mathrm{~h}$. As telomerase activities 
A

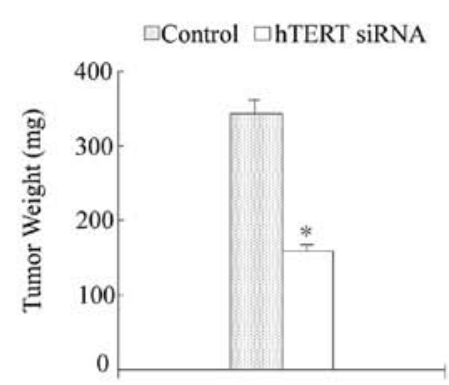

C

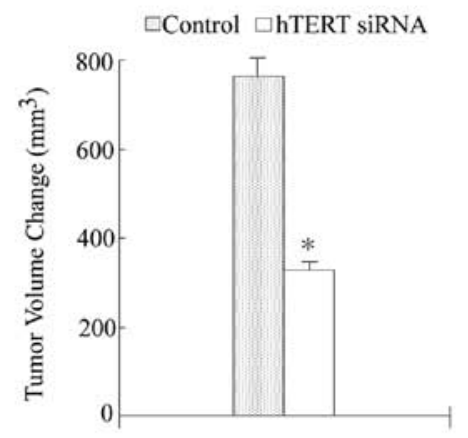

B

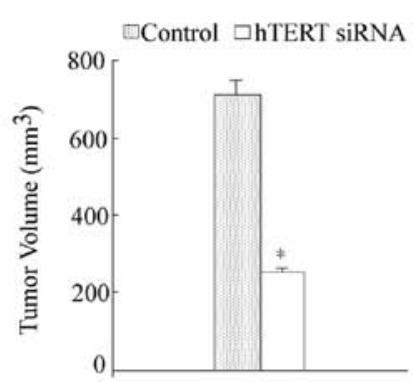

D

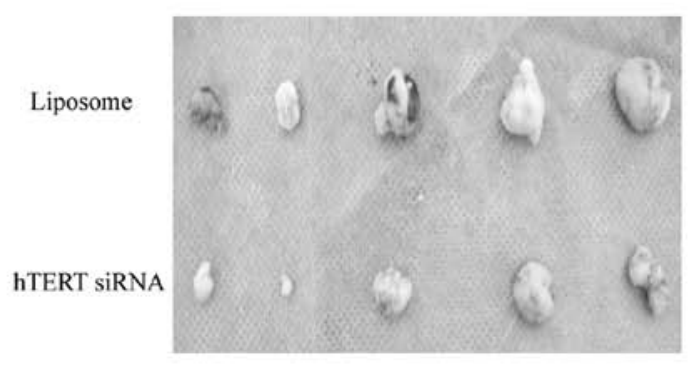

E

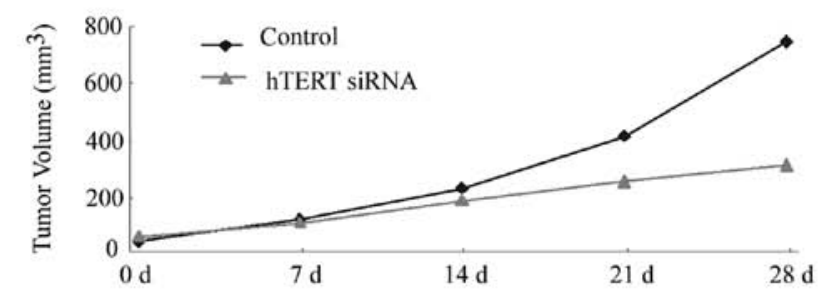

F
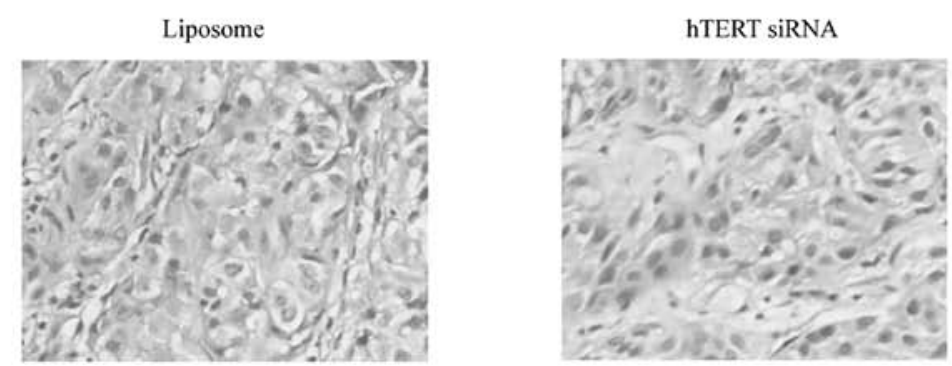

Figure 6. A549 cells $\left(5.0 \times 10^{6}\right)$ were injected into the flank of nude mice to bear tumors. After 3 weeks, visible tumors had developed at the injection sites. To determine the therapeutic effectiveness of hTERT siRNA, intratumoral treatments with hTERT siRNAs alone or plus Lipofectamine 2000 were started and repeated 2 times every week for a total of 4 weeks. (A) Xenograft weight $\left(\mathrm{t}=2.397,{ }^{*} \mathrm{P}<0.05\right)$. (B-E) Xenograft volumes $\left(\mathrm{t}=2.364,{ }^{*} \mathrm{P}<0.05\right)$ and volume changes $\left(\mathrm{t}=2.368,{ }^{*} \mathrm{P}<0.05\right)$. (F) Comparison of tumor histology.

were associated with the cell cycle, we further observed the effect of hTERT siRNA on the early apoptosis of lung adenocarcinoma cells by Annexin V-FITC/PI double-staining flow cytometry. There are several reports about the growth inhibitory effect of hTERT siRNA in cancer cells. Dong et al (26) transfected siRNA targeting hTERT into two breast cancer cell lines and the MTT assay showed a 50\% reduced cell viability in breast cancer cells within two days after transfection, while after three days of transfection the cell viability had an ascending trend, consistent with our study.

Collectively, our in vitro study indicates that hTERT siRNA can down-regulate the expression of hTERT mRNA and hTERT protein, inhibit telomerase activity and accordingly induce the apoptosis of lung adenocarcinoma A549 cells. The effect of hTERT siRNA on hTERT gene expression reached its maximum at $48 \mathrm{~h}$ and persisted $72 \mathrm{~h}$ after transfection. The silencing potency of hTERT siRNA in our study was lower than that reported in previous studies $(20,24,25)$. This difference may mainly be due to the siRNA transfection efficiency, siRNA properties and different cell lines. In our study, the siRNA synthesized by in vitro transcription had a small molecular weight but a high transfection efficiency, higher than $80 \%$ as reported, but labeling and simultaneously monitoring its transfection efficiency was not possible, which is a shortcoming of this approach. Furthermore, different cell lines can have distinct transfection efficiencies and silencing potencies. 
A

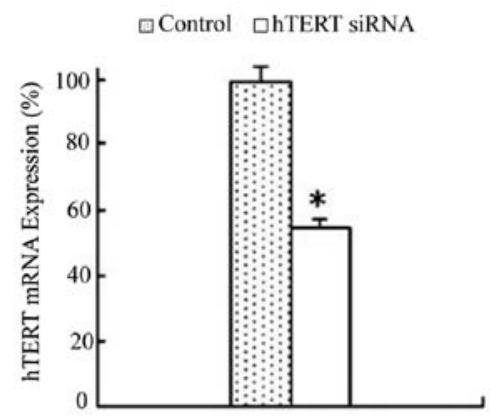

C

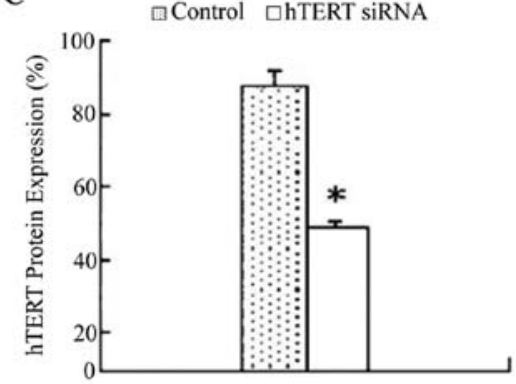

B

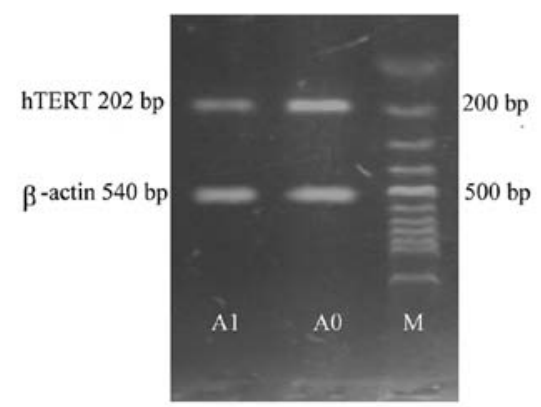

M: Marker A0: Liposome A1: hTERT siRNA
D

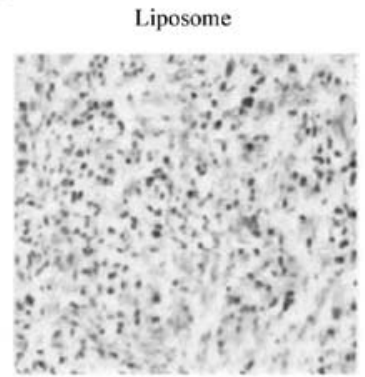

hTERT SIRNA

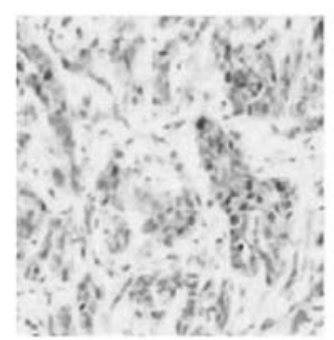

$\mathbf{E}$

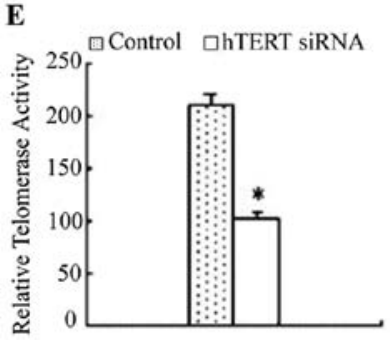

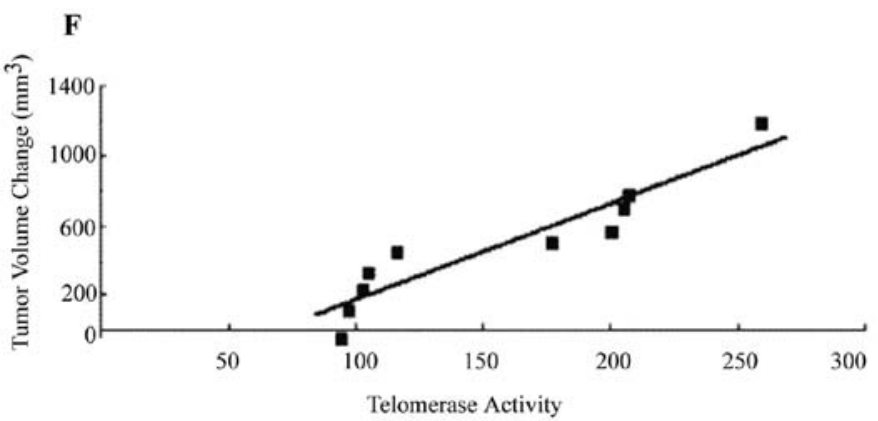

Figure 7. (A and B) hTERT mRNA expression of lung adenocarcinoma xenografts ( $\mathrm{t}=21.179$, $\left.{ }^{*} \mathrm{P}<0.05\right)$. (C and D) hTERT protein expression of lung adenocarcinoma xenografts $\left(\mathrm{t}=12.901,{ }^{*} \mathrm{P}<0.05\right)$. Nuclei of hTERT protein positive cells were stained by immunohistochemistry. (E) Telomerase activity of lung adenocarcinoma xenografts $\left(\mathrm{t}=7.727,{ }^{*} \mathrm{P}<0.05\right)$. (F) Correlation analysis between tumor volume change and telomerase activity $(\mathrm{r}=0.932, \mathrm{P}<0.05)$. The lower the telomerase activities of the xenografts, the slower the growth. Relative telomerase activity of the xenografts is positively correlated to tumor volume change.

Up to date, there are only a few studies on a xenograft model of lung adenocarcinoma in vivo using RNAi technology. Therefore, we further examined the inhibition of hTERT siRNA on the growth of lung adenocarcinoma xenografts. According to the results of the lung adenocarcinoma cell growth inhibition tests, we administered hTERT siRNA by injection into the xenograft tumor two times every week and measured its growth. Our study found that hTERT siRNA can significantly inhibit the growth of lung carcinoma xenografts and the inhibitory rate reached $64.28 \%$. It was implied that siRNA can resist the degradation of RNase and silence genes homologous to siRNA in vivo. Further analysis on the xenograft tumor tissue showed that hTERT siRNA alleviated the heteromorphism of the xenograft tumor tissue. The growth of the xenograft tumor was limited but necrosis was not apparent. We therefore, speculate that the growth inhibition of hTERT siRNA is different from the direct killing of tumor cells by traditional cytotoxic drugs. It is possible that inhibition of the growth of the xenograft tumor is due to inhibition of the proliferation of cancer cells and the induction of cell apoptosis.
Dong et al (26) transfected siRNA targeting hTERT into two breast cancer cell lines and the volume of the tumor in the experimental group was $40 \%$ of the control group. Tumor cells (20\%) showed apoptosis at $48 \mathrm{~h}$ after transfection while the control group showed no signs of apoptosis. The results of their study in a way support our speculation. To determine the relationship between the inhibition of tumor growth and the expression of hTERT mRNA, telomerase activity in vivo, we examined the expression of hTERT mRNA, hTERT protein and telomerase. Our results showed that hTERT siRNA significantly down-regulated the expression of hTERT mRNA, hTERT protein and telomerase activity. The analysis of correlation also revealed that the volume change of the xenograft was positively correlated with the telomerase activity. The results indicate that hTERT siRNA suppressed the growth of xenograft tumor in vivo by specific down-regulation of hTERT mRNA, hTERT protein expression and the inhibition of telomerase activity.

Our present data clearly show that down-regulation of telomerase expression and thus suppression of tumor growth 
by siRNA targeting hTERT is an attainable goal. Thus, one of our future objectives is to find a suitable way for this method to be used in clinical settings. Since some theoretical issues have already been raised concerning the safety of certain RNAimediated therapies (27), we may search for more efficient siRNAs and suitable vectors.

\section{Acknowledgements}

This study was supported by grant no. 07JJ3055 from Natural Science Foundation of Hunan, China.

\section{References}

1. Feng J, Funk WD, Wang SS, et al: The RNA component of human telomerase. Science 269: 1236-1241, 1995.

2. Harrington L, Zhou W, McPhail T, et al: Human telomerase contains evolutionarily conserved catalytic and structura subunits. Genes Dev 11: 3109-3115, 1997.

3.Djojosubroto MW, Choi YS, Lee HW and Rudolph KL: Telomeres and telomerase in aging, regeneration and cancer. Mol Cells 15: 164-175, 2003

4. Shay JW and Wright WE: Telomerase therapeutics for cancer: challenges and new directions. Nat Rev Drug Discov 5: 577-584 2006.

5. Hahn WC: Role of telomeres and telomerase in the pathogenesis of human cancer. J Clin Oncol 21: 2034-2043, 2003.

6. Chen Q, He J and Yi H: Study on telomerase activity and its clinical value in human non small cell lung cancer. Hunan Yi Ke Da Xue Xue Bao 26: 221-222, 2001 (In Chinese).

7. Tomlinson RL, Abreu EB, Ziegler T, et al: Telomerase reverse transcriptase is required for the localization of telomerase RNA to cajal bodies and telomeres in human cancer cells. Mol Biol Cell 19: 3793-3800, 2008.

5. Hahn WC: Role of telomeres and telomerase in the pathogenesis of human cancer. J Clin Oncol 21: 2034-2043, 2003.

8. Sung YH, Choi YS, Cheong C and Lee HW: The pleiotropy of telomerase against cell death. Mol Cells 19: 303-309, 2005.

9. Folini $\mathrm{M}$ and Zaffaroni $\mathrm{N}$ : Targeting telomerase by antisensebased approaches: perspectives for new anti-cancer therapies. Curr Pharm Des 11: 1105-1117, 2005.

10. Melana SM, Holland JF and Pogo BG: Inhibition of cell growth and telomerase activity of breast cancer cells in vitro by 3'-azido3'-deoxy thymidine. Clin Cancer Res 4: 693-696, 1998.

11. Bertrand JR, Pottier M, Vekris A, Opolon P, Maksimenko A and Malvy C: Comparison of antisense oligonucleotides and siRNAs in cell culture and in vivo. Biochem Biophys Res Commun 296: 1000-1004, 2002.
12. Brown DA, Kang SH, Gryaznov SM, et al: Effect of phosphorothioate modification of oligodeoxynucleotides on specific protein binding. J Biol Chem 269: 26801-26805, 1994.

13. Gewirtz AM: On future's doorstep: RNA interference and the pharmacopeia of tomorrow. J Clin Invest 117: 3612-3614, 2007.

14. Gondi CS and Rao JS: Concepts in in vivo siRNA delivery for cancer therapy. J Cell Physiol 220: 285-291, 2009.

15. Filipowicz W: RNAi: the nuts and bolts of the RISC machine. Cell 122: 17-20, 2005.

16. Rivas FV, Tolia NH, Song JJ, et al: Purified Argonaute2 and an siRNA form recombinant human RISC. Nat Struct Mol Biol 12: 340-349, 2005.

17. Reynolds A, Leake D, Boese Q, Scaringe S, Marshall WS and Khvorova A: Rational siRNA design for RNA interference. Nat Biotechnol 22: 326-330, 2004.

18. Ui-Tei K, Naito Y, Takahashi F, et al: Guidelines for the selection of highly effective siRNA sequences for mammalian and chick RNA interference. Nucleic Acids Res 32: 936-948, 2004.

19. Elbashir SM, Harborth J, Lendeckel W, Yalcin A, Weber K and Tuschl T: Duplexes of 21-nucleotide RNAs mediate RNA interference in cultured mammalian cells. Nature 411: 494-498, 2001.

20. Xia Y, Lin RX, Zheng SJ, et al: Effective siRNA targets screening for human telomerase reverse transcriptase. World J Gastroenterol 11: 2497-2501, 2005.

21. Wu ZQ, Guo QL, You QD, Zhao L and Gu HY: Gambogic acid inhibits proliferation of human lung carcinoma SPC-A1 cells in vivo and in vitro and represses telomerase activity and telomerase reverse transcriptase mRNA expression in the cells. Biol Pharm Bull 27: 1769-1774, 2004.

22. Takei Y, Kadomatsu K, Yuzawa Y, Matsuo S and Muramatsu T: A small interfering RNA targeting vascular endothelial growth factor as cancer therapeutics. Cancer Res 64: 3365-3370, 2004.

23. Schwarz DS, Hutvagner G, Du T, Xu Z, Aronin N and Zamore PD: Asymmetry in the assembly of the RNAi enzyme complex. Cell 115: 199-208, 2003.

24. Kosciolek B, Kalantidis K, Tabler M and Rowley PT: Inhibition of telomerase activity in human cancer cells by RNA interference. Mol Cancer Ther 2: 209-216, 2003.

25. Shammas MA, Koley H, Batchu RB, et al: Telomerase inhibition by siRNA causes senescence and apoptosis in Barrett's adenocarcinoma cells: mechanism and therapeutic potential. Mol Cancer 4: 24, 2005.

26. Dong X, Liu A, Zer C, et al: siRNA inhibition of telomerase enhances the anti-cancer effect of doxorubicin in breast cancer cells. BMC Cancer 9: 133, 2009.

27. Frantz S: Safety concerns raised over RNA interference. Nat Rev Drug Discov 5: 528-529, 2006. 